\title{
Aspectos metodológicos clave para la formación de futuros maestros de Educación Física Key methodological aspects for the training of future Physical Education teachers
}

Daniel Bores García, Antonio Luis Marín Rojas, César Méndez Domínguez, Juan José Mijarra Murillo, José Manuel Delfa De La Morena

Universidad Rey Juan Carlos (España)

Resumen. El presente artículo trata de ofrecer la visión consensuada y compartida desde el Área de Educación Física y Deportiva de la Universidad Rey Juan Carlos acerca de cuáles deben ser los elementos esenciales del abordaje del proceso formativo de los futuros maestros de Educación Física. Entre ellos se exponen las claves sobre la importancia de la motricidad, el rol del alumno en las clases teóricas y prácticas, que debe ser activo y reflexivo en todo momento, la comunicación docente-discente, el trabajo en grupos como herramienta de interacción social y co-aprendizaje, las tareas individuales desde una perspectiva crítica y los aspectos más relevantes sobre el proceso de programación de los contenidos de Educación Física, tales como la secuenciación, el abordaje global de los contenidos y el uso de las nuevas tecnologías. Por último, se expone el consenso de los docentes del área sobre los objetivos, contenidos y procedimientos evaluativos de las asignaturas del área, tomando como referencia la materia de Didáctica de la Educación Física del Grado de Maestro en Educación Primaria con Mención en Educación Física.

Palabras clave: Educación Física, formación inicial, enseñanza universitaria, educación formal, plan de estudios.

Abstract. The present article aims at offering a consensual and shared vision from the Area of Physical Education and Sports at Rey Juan Carlos University about what the essential elements of the approach to the training process of future Physical Education teachers should be. Among others, we explain the key factors on the importance of motor skills, the role of students in theoretical and practical classes, which must be active and reflective at all times, teacher-student communication, work in groups as a tool for social interaction and colearning, individual tasks from a critical perspective, as well as the most relevant aspects of the process of planning Physical Education contents, such as sequencing, global approach to content, and the use of new technologies. Finally, the consensus of teachers from this area on objectives, contents, and subject evaluation procedures is described, taking the subject of Physical Education Didactics from the Degree of Primary Education with Mention in Physical Education as a reference.

Key words: Physical Education, initial training, university education, formal education, curriculum.

\section{Introducción}

Han pasado ya seis años desde la primera promoción de graduados en Magisterio de Primaria con la mención en Educación Física. Durante este tiempo, la comunidad universitaria ha asistido en nuestro país a un progresivo deterioro de los recursos existentes tanto para el profesorado como para el alumnado (Martínez, 2016) que ha traído consigo dificultades para aumentar o, siquiera, mantener la calidad de la enseñanza universitaria (Parellada, 2015). No obstante, hay estudios que muestran la existencia de buenas prácticas que han logrado paliar los efectos de la crisis y han contribuido a la creación y desarrollo de programas formativos de calidad en las enseñanzas superiores (Gutiérrez et al., 2018; López-Pastor \& Pérez-Pueyo, 2017; Vallejo \& Molina, 2014). Siguiendo a Gutiérrez et al. (2018), el papel del profesorado, entre otros, es crucial para la consecución de una enseñanza universitaria de calidad. En el presente artículo optamos por poner el foco en los aspectos metodológicos que consideramos claves en la formación inicial de docentes de Educación Física, constituyendo estos el núcleo de la práctica docente de los miembros del área de Educación Física y Deportiva que imparten clase en las diferentes asignaturas de los Grados de Primaria e Infantil en la Universidad Rey Juan Carlos.

La presencia de una Didáctica de la EF está justificada con la nueva reforma Educativa ante la necesidad de una formación de especialistas en Educación Física, para proporcionar conocimientos sobre los objetivos y contenidos, así

Fecha recepción: 12-02-19. Fecha de aceptación: 08-09-19

Daniel Bores García

dboresgarcia@gmail.com como su metodología y evaluación. La búsqueda de formas de enseñar coherentes es todavía una cuestión objeto de controversia constante, en la que es necesaria una profunda reflexión que desarrolle un proceso de humanización por parte de todos los agentes implicados. Las actividades de aprendizaje en EF se siguen diseñando dentro de un contexto sociocultural determinado, conectando los conceptos de enseñar y aprender, considerando que las situaciones educativas de carácter corporal representan una acción educativa, y en donde se trata de justificar todos los conocimientos que integran la Educación Física y su tratamiento didáctico, en una especie de cajón de sastre (Contreras, 1998).

La metodología en torno a la que giran las asignaturas de la Mención en Educación Física del Grado en Educación Primaria en la Universidad Rey Juan Carlos (URJC en adelante) encuentra sus cimientos en una serie de principios de actuación docente y estrategias didácticas fruto del intercambio dialógico y el consenso entre el personal del área de Educación Física y Deportiva de esta universidad, que pasaremos a detallar a continuación en los siguientes epígrafes.

El rol de la motricidad en el proceso de enseñanza-aprendizaje de la Educación Física en el contexto universitario

Antes de dar respuesta a la pregunta, conviene situarnos en la relación de la Educación Física (de ahora en adelante EF) con la motricidad en el ámbito educativo. «La asignatura de Educación Física en la Educación Primaria tiene como principales objetivos el desarrollo de las capacidades motrices, la adquisición de hábitos saludables y de conducta y la práctica de actividades físicas, deportivas y artísticas. La Educación Física contribuye a la educación en el cuidado 
de la salud y de la seguridad a través del conocimiento del propio cuerpo.» (BOCM 175, del 25 de julio de 2014). El rol que desempeña la motricidad en Educación Física es muy importante, buscando en todo momento el aprendizaje de estrategias para ayudar al alumnado a desarrollarse motrizmente. Como referencia teórica, se utiliza la Taxonomía de Harrow (1972), en la que se presentan diferentes acciones motrices en función del nivel de habilidad psicomotriz, desde lo más simple o adquirido por el desarrollo natural del niño a lo más complejo, puesto que es necesario adquirir los niveles inferiores para llegar a los niveles superiores de dicho aprendizaje motor (Harrow, 1972).

En el área curricular de EF se busca un desarrollo integral del individuo, por lo que no solamente se da importancia al aspecto motor, sino también al afectivo, social y cognitivo, que se relaciona con el movimiento y con las relaciones del alumno con su entorno. Por esto, en nuestras clases de EF, se otorga también una gran importancia al ofrecimiento de recursos y estrategias que ayuden al alumnado a resolver situaciones que se les presenten como futuros maestros, puesto que el aprendizaje de contenidos o de habilidades motrices pueden adquirirse en cualquier momento y no se necesitan grandes destrezas de movimiento para desempeñar el rol de profesor, por lo que nuestro aprendizaje se centra en la didáctica; es decir, en cómo se pueden transmitir dichos contenidos y cómo pueden ayudar a sus futuros alumnos en el desarrollo motor y a que adquieran diferentes habilidades necesarias para su formación integral, todo ello mediante el uso del juego como principal herramienta didáctica, destacando la necesidad de dar ejemplo en base a la actitud ante la EF y promover no solo la práctica de la misma, sino su importancia en la vida diaria y en nuestra sociedad.

\section{El rol del alumnado y del profesor en las clases teóricas y prácticas}

Desde el área de Educación Física y deportiva de la URJC y en consonancia con una perspectiva constructivista del aprendizaje, se pretende que el alumno sea un sujeto activo el mayor número de horas lectivas posible, tanto clases teóricas como prácticas. Una metodología activa, de acuerdo con García, Urionabarrenetxea y Bañales (2016) busca responsabilizar al estudiante de su proceso de enseñanza, confiriéndole un papel protagonista frente al método expositivo tradicional. Esta visión se materializa, en la línea de lo que expone Eirin (2018, p.261), a través de una figura docente caracterizada por proporcionar oportunidades e incentivos a los alumnos para que construyan su propio conocimiento, en lugar de «proporcionárselo» o «impartírselo». La estructura de las clases teóricas diseñadas en esta área se articula en forma de taller de creación grupal, donde a los discentes se le plantea un reto o una incógnita al inicio de la clase, en relación al temario de la asignatura, que ellos deben resolver in situ trabajando en pequeños grupos, antes de la finalización de esta. Este desafío cognitivo y afectivo, planteado como una tarea que requiere de pensamiento, análisis y puesta en común, pretende impulsar el compromiso e implicación del alumnado, ya que, como afirman Tomlinson y Strickland (2005), Shernoff et al., (2016) y Strati et al., (2017) citados por Ochoa (2018), los estudiantes apren- den en mayor medida cuando el trabajo es moderadamente retador y cuando perciben el apoyo necesario para realizar lo que inicialmente parece fuera de su alcance. Para ello, de forma habitual, hacen uso de equipos informáticos con acceso a internet para documentarse o consultar cualquier aspecto relativo a la tarea. El papel del docente en estas clases se configura como un guía, que contextualiza la tarea a realizar y orienta el proceso, pero no da las respuestas. En consonancia con el pensamiento de Martínez (1995), el profesor será el organizador de la interacción de los alumnos con el conocimiento. Al final de la clase, se realiza una composición grupal con las aportaciones de los alumnos, dirigida por el docente, donde se destacan los elementos más relevantes de la tarea propuesta, sirviendo de feedback compartido al trabajo realizado durante dos horas.

El desarrollo de las clases prácticas se organiza en dos bloques diferenciados por el protagonismo de los distintos agentes educativos. En la primera mitad de las prácticas, es el docente el que selecciona y dirige las actividades a realizar, donde los alumnos actúan como participantes en estas. Cada sesión práctica está enfocada en el tratamiento, de forma monográfica, de un bloque de contenidos concreto en consonancia con los descritos en el currículo oficial de la Educación Física en la Comunidad de Madrid (decreto 89/ 2014 de 24 de julio, del Consejo de Gobierno, por el que se establece para la Comunidad de Madrid el Currículo de la Educación Primaria.). En el segundo bloque de prácticas, es el alumnado, organizado en subgrupos, el que dirige las sesiones prácticas de trabajo en función a un bloque de contenidos asignado por el profesor (en relación con la legislación educativa vigente) y como parte de la evaluación de sus competencias en el diseño e implementación de procesos de enseñanza-aprendizaje en el área de Educación Física. En esta parte, el papel del docente es de observador, recogiendo información del desempeño práctico del alumnado en la dirección de sesiones y realizando un feedback al final de cada sesión de trabajo, en forma de intercambio, como experiencia de aprendizaje.

Las actividades a desempeñar tanto en las sesiones prácticas dirigidas por el profesor, como en aquellas dirigidas por los alumnos, se articulan alrededor del juego como recurso didáctico capaz de realizar múltiples aportaciones pedagógicas en el área de Educación Física: ser una actividad altamente motivante, cooperar en el desarrollo del conocimiento o facilitar el aspecto social y la coeducación (Del Valle \& De la Vega, 2007).

\section{La comunicación entre profesor y alumno: el feedback}

El proceso de retroalimentación del desempeño de los educandos (y del docente) se instala en la metodología del área de Educación Física y Deportiva de la URJC como un elemento integrado en la práctica educativa diaria.

Nuestro concepto de feedback no se concibe como un producto final, sino como un proceso conectado de forma esencial y continua con las tareas. No existe propuesta o trabajo discente sin feedback. La razón de implementar esta forma de retroalimentación responde a la aplicabilidad del feedback en futuros procesos de construcción. Siguiendo a Carless (2016, p.17), «uno de los principales problemas que 
los estudiantes perciben en relación al feedback es que, a menudo, les llega demasiado tarde para poderlo utilizar, especialmente si los comentarios son sumativos y en relación a evaluaciones realizadas al final de semestre». Por tanto, la decisión de integrar ciclos de orientación y feedback dentro de la programación de la asignatura puede identificarse como una estrategia didáctica útil (Hounsell et al., 2008).

En relación a la visión constructivista de la didáctica de la Educación Física anteriormente mencionada, tanto en las tareas desarrolladas respecto de contenidos teóricos como prácticos, se favorece un feedback no centrado en exclusividad en los errores del alumnado. Plantear un feedback en positivo, enfocado a reorientar los procesos de enseñanzaaprendizaje, favoreciendo el intercambio profesor-alumno y alumno-alumno puede ayudar a aumentar la motivación de estos hacia las unidades de aprendizaje tratadas. Además, y en consonancia con Carless (2016), un feedback exitoso puede depender en gran medida del establecimiento de una relación positiva entre los participantes de este.

El proceso de retroalimentación, según nuestra concepción del mismo, se conforma como un diálogo entre los agentes educativos, donde también tienen cabida la autorregulación y el feedback entre iguales y se contribuye no solo a evitar posibles errores sino a identificar los caminos para superarlos. El feedback no solo está enfocado a mejorar el desempeño inmediato de los alumnos en las tareas académicas, sino también, como afirman Boud y Molloy (2015), para impulsar la capacidad de los educandos de construir su propia capacidad de autorregularse y autosupervisarse en futuros entornos profesionales donde puede no ser común contar con procesos de aprendizaje estructurados. Por otra parte, favorecer el feedback entre iguales posibilitando la percepción de valoraciones de compañeros puede contribuir a aumentar la comprensión, la familiarización y la responsabilidad en las tareas (Hortigüela et al, 2015).

\section{El trabajo en grupos como clave metodológica}

El hecho de que al menos el 50\% de las pruebas evaluables en las asignaturas impartidas por el área de Educación Física y Deportiva de la URJC lo constituyan tareas grupales puede dar una visión aproximada de la importancia que desde este área se le pretende dar al desarrollo de las competencias sociales de los alumnos del Grado de Educación Primaria. Desde nuestra perspectiva, y en la línea de lo que defiende Maldonado (2007, p.265), «el aprendizaje se presenta como un proceso social que se construye en la interacción no sólo con el profesor, sino también con los compañeros, con el contexto y con el significado que se le asigna a lo que se aprende».

Este trabajo en equipo no se limita al espacio de aula ordinaria, sino que trasciende en nuestras asignaturas fuera del horario lectivo. Dentro de la programación de cada asignatura, se establecen tareas de forma periódica que requieren de una labor de documentación autónoma previa al tratamiento de los contenidos en clase, con el fin de poder dedicar más horas presenciales a procesos de construcción grupal, debates o resolución de dudas que, en definitiva, pueden favorecer el desarrollo de actitudes de responsabili- dad y una mayor implicación del alumnado en clase (Mérida et al, 2011).

Las contribuciones del trabajo colaborativo en entornos de educación superior han sido ampliamente reportadas en la literatura científica actual. Gutiérrez et al. (2011), Escribano, (1995) y Brown y Atkins, (1988), citados por Vargas et al (2016, p. 71) afirman que «esta estrategia metodológica puede desarrollar diferentes aprendizajes en los estudiantes en los aspectos cognitivos (análisis, razonamiento lógico, valoraciones y juicios, pensamiento crítico, síntesis, diseño, aplicación de estrategias a contextos diferentes, resolución de problemas, etc.) y en lo afectivo y social (responsabilidad, desarrollo de la autoestima, autorregulación del aprendizaje, trabajo con otros, etc.) Además, Ibarra y Rodríguez (2007) sugieren que este tipo de organización es valorado positivamente por el alumnado ya que les facilita la expresión de opiniones y argumentos, y poner en valor las propias aportaciones y las de los compañeros.

\section{Las tareas individuales: aprendiendo desde una pers- pectiva crítica, reflexiva y autónoma}

A pesar de las contribuciones que se desprenden del trabajo en grupo en entornos de educación universitaria y sus múltiples beneficios, parece razonable pensar que otros tipos de organización del alumnado también podrían colaborar en el desarrollo de la formación integral de estos y sus competencias como futuros profesionales en el ámbito educativo. De acuerdo con González (2013, p.7), «la situación óptima para el aprendizaje es aquella en la que se pueden movilizar, combinar y utilizar estrategias, recursos y oportunidades diversas, reconociendo la diversidad de comportamientos y capacidades en respuesta a múltiples situaciones individuales y contextuales».

Además de las tareas grupales anteriormente mencionadas en las asignaturas impartidas por el área de Educación Física y Deportiva de la URJC, el trabajo individual se inserta en la metodología de esta área como un recurso para optimizar los aspectos relativos al autoaprendizaje, la autonomía, la capacidad crítica, la creatividad o la reflexión atendiendo en la medida de los posible a las características intrapersonales del alumnado (tanto cognitivas como afectivas). Las tareas individuales nos permiten focalizarnos en el nivel demostrado de aptitudes y competencias que, desde la perspectiva del trabajo en conjunto, son complejas de valorar. Es el caso del dominio básico del bloque teórico de las asignaturas, de las habilidades comunicativas y de relación socio-afectiva con el alumnado en la dirección de sesiones o de la capacidad de estos para liderar, gestionar y organizar grupos y recursos materiales.

Dentro de las líneas metodológicas de esta área dirigida al diseño e implementación de situaciones de enseñanzaaprendizaje de carácter individual para la formación de los futuros maestros, cobra especial sentido el concepto «autonomía». Las secuencias didácticas planteadas en clase teórica, como se indicó anteriormente, deben ir precedidas de un trabajo autónomo de cada alumno en la labor de búsqueda, selección y análisis de información relevante al respecto de los contenidos de las asignaturas, imprescindible para afrontar con garantías los retos planteados en el aula pre- 
sencial y contribuir al alcance de los objetivos del grupo de trabajo. Estas estrategias en la introducción de tareas individuales pretenden, en la línea de lo que expone Cabeza (2011, pág. 11), implicar la conciencia, gestión y control de las propias capacidades y conocimientos de los discentes desde un sentimiento de competencia o eficacia personal, considerando también la capacidad de autoevaluarse, el pensamiento estratégico y el manejo eficiente de un conjunto de recursos educativos.

Estructuración de las asignaturas específicas de EF en el Grado de Educación Primaria con mención en EF de la URJC

Son cinco las asignaturas relacionadas con la EF que existen en el Grado de Educación Primaria con mención en EF en la URJC. Una de ellas, llamada «Introducción a la Educación Física» es obligatoria para todo alumnado aunque no curse la mención de EF, impartiéndose en el segundo curso del grado. Las cuatro asignaturas restantes son específicas y de necesaria realización si se quiere obtener la mención de EF. Estas cuatro asignaturas específicas se denominan «Didáctica de la Educación Física», «Expresión Corporal», «Motricidad y Capacidades Físicas» e «Iniciación Deportiva». Desde el área de EF de la URJC se ha tenido una perspectiva global y consensuada en la elaboración de estas cinco asignaturas acordando unas bases comunes que se detallan a continuación.

- Objetivos: los objetivos de cada una de las cinco asignaturas van en concordancia con la asimilación de las competencias específicas de cada una de ellas, de manera que el alumnado adquiera los recursos, conocimientos y habilidades suficientes para su desempeño. Amodo de ejemplo, y dado que este artículo versa fundamentalmente sobre aspectos metodológicos claves para la formación de los futuros maestros de EF, los objetivos específicos de la asignatura «Didáctica de la Educación Física» son los siguientes:

Conocer el marco normativo (currículo oficial) de la Educación Primaria y sus implicaciones en la labor de programación a nivel estatal y autonómico (Comunidad de Madrid)

Identificar las metodologías más representativas en el ámbito de la Educación Física y sus implicaciones didácticas.

Desarrollar dominio en el diseño y adaptación de elementos curriculares de una programación a un contexto concreto.

Desarrollar competencia en el desarrollo e implementación de unidades didácticas y sesiones de trabajo.

Adquirir competencia en el diseño de procesos de evaluación en el área de Educación Física.

- Potenciar aptitudes de relación social en pos de construcciones o proyectos grupales.

- Desarrollar competencia, autonomía y autogestión en proyectos individuales.

- Contenidos: los contenidos de las 5 asignaturas son de carácter teórico práctico, ya que en las «clases teóricas» se realizan ejercicios prácticos y en las «clases prácticas» se comentan aspectos teóricos.

En la asignatura «Introducción a la EF» (obligatoria para todo el alumnado, siendo la primera materia sobre EF que cursan en el grado), los contenidos se basan fundamentalmente en la importancia de la EF, el juego como herramienta, la atención a la diversidad y la educación en valores. En esta asignatura se realizan 4 prácticas (niveles 3,4,5 y 6 de la Taxonomía de Harrow) las cuales se profundizarán en cada una de las cuatro asignaturas específicas de EF que han de realizar los alumnos para obtener la mención.

En la asignatura «Didáctica de la EF» los contenidos versan sobre el sistema educativo, uso de TICs, investigación en EF y en el proceso de enseñanza-aprendizaje. Se realizan cuatro prácticas sobre habilidades perceptuales (nivel 3 de Harrow): percepción corporal, habilidades motrices básicas, equilibrio y lateralidad y percepción espacio temporal.

En la asignatura «Motricidad y Capacidades Físicas» se incluyen nociones de anatomía, biomecánica, fisiología, actividades físicas en el medio natural y hábitos de vida saludables. Se realizan cuatro prácticas sobre capacidades físicas (nivel 4 de Harrow): fuerza, resistencia, velocidad y flexibilidad.

- En la asignatura «Iniciación Deportiva» los contenidos fundamentales se basan en la estructura y función del deporte, modelos de enseñanza, aprendizaje motor, deporte, sociedad y educación. Se realizan cuatro prácticas sobre destrezas motrices (nivel 5 de Harrow): deportes individuales con y sin oposición y deportes colectivos con y sin oposición.

En la asignatura «Expresión Corporal» los contenidos versan sobre el cuerpo y el movimiento como herramienta de expresión, la danza, las artes circenses y la dramatización.

- Evaluación: la evaluación es continua y formativa en las cinco asignaturas impartidas desde el área, existiendo además pruebas comunes a todas ellas, que podrán diferir en la ponderación y obviamente en los contenidos. Todas las asignaturas tienen en común las siguientes pruebas de evaluación:

Prueba escrita de carácter teórico práctico que puede consistir en preguntas tipo test, preguntas cortas, pregunta a desarrollar o una combinación de ambas, opción esta última la más elegida por el profesorado al considerar que evalúa mejor el aprendizaje del alumno de los contenidos tratados.

Exposición y/o trabajos grupales que, según la asignatura, pueden ser exposiciones orales con soporte audiovisual, debates por grupos, elaboración de sesiones y unidades didácticas, creación de blogs o montaje acrocoreográfico.

Exposición individual en la que el alumno deberá asumir el rol de profesor y realizar una actividad en la que el resto de sus compañeros adquieran el rol de alumno. En esta prueba se valoran cinco apartados: la creatividad y ludicidad de la actividad, la organización y control de la clase, la comunicación técnica y relación socio-afectiva, el tiempo de actividad motriz efectiva (TAME) y la capacidad de adaptarse a situaciones imprevistas. Destacamos este último apartado 
como algo innovador y muy significativo en la formación del alumno para su desempeño como futuro profesor, ya que, gracias a este ítem, desarrollan su plasticidad y se les inicia en la resolución de conflictos que aparecen diariamente en el aula.

\section{Evaluación y contenidos de la asignatura de Didáctica de la Educación Física}

El abordaje de la evaluación en la asignatura Didáctica de la Educación Física impartida desde el área Educación Física y Deportiva de la URJC, contempla, como se ha mencionado con anterioridad, la valoración del desempeño del alumnado en tareas individuales y grupales. La ponderación del conjunto de pruebas individuales (prueba escrita sobre contenidos teóricos y examen práctico individual) y grupales (creación de unidad didáctica y trabajo de iniciación a la investigación en Educación Física) se configura con un reparto equilibrado entre ambas (55\% - 45\% de la puntuación final respectivamente). A continuación, y con el propósito de clarificar las características de cada prueba se expresará una síntesis de estas:

Las pruebas individuales se componen de dos tareas. Por un lado, y al final del periodo lectivo, se administra una prueba escrita, configurada en torno a una serie de preguntas cortas y de desarrollo sobre los contenidos teóricos de la asignatura. Para superar la prueba los alumnos deberán mostrar un dominio de los contenidos tratados en clase suficiente para conseguir al menos el 50\% de la puntuación asignada a la prueba (30\% de la ponderación total de la asignatura). La segunda prueba consiste en un examen práctico individual (25\% de la ponderación total de la asignatura) donde el alumnado dirigirá una actividad práctica, en relación con el bloque práctico de la asignatura que se le habrá asignado, dirigiendo al resto de alumnos de clase como «niños de Educación Primaria». El tiempo de la prueba oscila entre 5 y 10 minutos por alumno valorándose su competencia demostrada respecto de 5 bloques de aptitudes prácticas: la ludicidad y creatividad del juego diseñado (20\% ponderación de la prueba), la relación socio-afectiva demostrada con el alumnado (20\% ponderación de la prueba), la organización y control del grupo de clase (20\% ponderación de la prueba), el tiempo de actividad motriz efectiva (TAME) desarrollado (20\% ponderación de la prueba), y la capacidad para adaptarse a situaciones imprevistas durante el desarrollo del juego (20\% ponderación de la prueba).

Las pruebas grupales también están representadas por dos tareas. La primera de estas se configura alrededor de la creación de una unidad didáctica o secuencia de aprendizaje (25\% ponderación total asignatura) precedida de los apartados que debe incluir una programación didáctica según la resolución de 26 de abril de 2017, de la Dirección General de Recursos Humanos por la que se convoca procedimiento selectivo para ingreso en el Cuerpo de Maestros y para la adquisición de nuevas especialidades en la Comunidad de Madrid. Esta expresión de objetivos didácticos, contenidos, metodología, evaluación y conjunto de sesiones de trabajo deberá ajustarse a un contexto social concreto libremente escogido por los distintos grupos de alumnos. Por otra parte, se establece una segunda prueba grupal que con- siste en la realización de una revisión bibliográfica en torno a un tema de interés y actualidad en el área de Educación Física escolar (20\% ponderación total asignatura). Para una dotar al alumnado de una operativa de actuación rigurosa respecto de la búsqueda bibliográfica se realizan en clase teórica una serie de seminarios de formación que tienen como objetivo adquirir competencia básica en la búsqueda, selección y análisis de evidencias científicas en bases de datos.

Los contenidos tratados en Didáctica de la Educación Física se establecen en torno al clásico binomio en materia educativa de teoría-práctica. Los contenidos teóricos se organizan en cuatro bloques temáticos:

a) La Educación Física en el sistema educativo, donde se trata la normativa educativa vigente al respecto de esta área a nivel estatal y en la Comunidad de Madrid.

b) El proceso de enseñanza-aprendizaje en la Educación Física: el currículo de Educación Física en Educación Primaria, orientado al desarrollo de los elementos curriculares principales de una programación didáctica.

c) El alumnado de Educación Primaria, acerca de las características cognitivas, sociales y motrices del alumnado entre 6 y 12 años.

d) La investigación en Educación Física, donde se atienden nociones básicas relativas a la metodología de la investigación en Educación Física.

Los contenidos prácticos, al igual que los anteriores, se organizan en cuatro bloques temáticos, en torno a los que se articula el establecimiento de las secuencias didácticas materializadas en talleres monográficos sobre:

a) Percepción corporal: actividades relativas a localización de las partes del cuerpo, estados de tensión-relajación, actitud postural y hábitos correctos, respiración y discriminación sensorial.

b) Percepción espacio temporal: actividades relativas a la situación y orientación en el espacio, el tratamiento de las distancias, las medidas, las formas, los trazados, la duración, ordenación y secuenciación de sucesos, la velocidad, los ritmos y las estructuras rítmicas

c) Equilibrio y lateralidad: actividades relativas a situaciones de inestabilidad donde se manifieste la necesidad del equilibrar el cuerpo en estático o en movimiento. Tratamiento de la lateralidad manual y pédica.

d) Habilidades motrices básicas: actividades relativas al dominio de habilidades como el desplazamiento, el giro, el salto, los lanzamientos y las recepciones.

La programación: aprendiendo a enseñar de forma estructurada y progresiva

Como defienden Gimeno y Pérez (1989), programar o planificar la enseñanza es algo imprescindible como futuros docentes, puesto que es necesario establecer un puente o nexo de unión entre la legalidad y la práctica docente, en el que la improvisación no sea protagonista y podamos anticiparnos a la mayor parte de situaciones en el aula. La labor de realizar una programación didáctica en base al currículo educativo oficial y adaptada al contexto concreto del centro y alumnado constituye uno de los cometidos nucleares dentro de las responsabilidades que ostenta el maestro de Educación Primaria. Tanto es así, que la actual Ley Orgánica 8/ 
2013, de 9 de diciembre, para la mejora de la calidad educativa (LOMCE en adelante), en su artículo 91 sobre las funciones del profesorado, estipula como primera función de estos «la programación y la enseñanza de las áreas, materias y módulos que tengan encomendados.»

Sin embargo, a pesar del sobrado conocimiento instalado en entornos educativos y profesionales acerca de la relevancia de desarrollar en los futuros maestros de Educación Física su competencia en programación, con la aparición de las menciones pertenecientes a los nuevos grados en educación primaria y la extinción de los magisterios de especialidad, las horas lectivas dedicadas a la didáctica de la Educación Física, y en concreto, a tareas específicas de programación, se han visto sensiblemente reducidas. Ante esta situación, la planificación y selección de los contenidos a tratar en las asignaturas de mención del área de Educación Física actuales parece convertirse en una tarea especialmente relevante que requiere de una sosegada reflexión.

Desde la plena consciencia de la coyuntura actual en la que se está desarrollando la formación de los maestros de mención, desde el área de Educación Física y Deportiva de la URJC, se plantean, a continuación, las líneas maestras que dirigen los procesos didácticos en torno a la tarea de programar en esta área en la etapa de Educación Primaria:

1. Diseñar secuencias de enseñanza-aprendizaje en contacto con la realidad legislativa vigente.

Desde la perspectiva de esta área, contextualizar las secuencias didácticas en la normativa educativa vigente en la comunidad de Madrid busca implementar prácticas de enseñanza enfocadas a optimizar la aplicabilidad de los aprendizajes, reduciendo la distancia entre la formación académica y futuro el desarrollo profesional de los alumnos. Asimismo, y consonancia con Medina et al (2010), con el fin de evitar prácticas de enseñanza excesivamente tecnificadas y descontextualizadas, parece necesario la adopción de estrategias metodológicas como integrar la teoría y la práctica en las actividades, desarrollar hábitos de formación permanente a partir de la formación inicial o integrar los componentes disciplinarios y pedagógicos del conocimiento profesional en la enseñanza universitaria.

2. Evitar una visión parcial del espectro de las metodologías docentes y sus beneficios y limitaciones.

No pocos autores han sugerido clasificaciones al respecto de los métodos docentes en los últimos años, también en el ámbito de la formación universitaria, establecidos según diferentes criterios taxonómicos (Rodriguez, 2011). Ante esta realidad, puede resultar compleja la tarea de dilucidar qué métodos didácticos pueden adaptarse mejor a una determinada realidad educativa en centros escolares, sobre todo si no se cuenta con la experiencia necesaria para realizar tales juicios. El propósito del área de Educación Física y Deportiva de la URJC a este respecto es tratar de ofrecer al alumnado una visión rigurosa y objetiva de las metodologías actuales más representativas con aplicabilidad en contextos de Educación Primaria, con especial énfasis en aquellas que posean técnicas y estrategias que favorezcan el desarrollo de los aspectos cognitivos, afectivos y motrices de los niños entre 6 y 12 años. Después de todo, la formación integral de los alumnos representa, según el artículo 16.2 de la actual LOMCE, una de las finalidades más relevantes de la etapa de
Educación Primaria.

3. Integrar el uso de las TICs en las secuencias didácticas presenciales y no presenciales.

Tras la implantación del Espacio Europeo de Enseñanza Superior (EEES) y gracias a las nuevas tecnologías de información y la comunicación (TICs) y las posibilidades de las innovaciones tecnológicas con aplicación en el ámbito educativo, se están produciendo una serie de cambios en la forma de enfocar los procesos de enseñanza aprendizaje, tanto para docentes como para los estudiantes universitarios. El uso de las TICs, para el área de Educación Física y Deportiva de la URJC representa un recurso imprescindible integrado en el desarrollo diario de la docencia a través de una triple funcionalidad, en relación con la enumeración propuesta por Ferro et al (2009): como recurso didáctico (soporte de presentaciones de contenidos y tareas, plataforma de desarrollo de blogs educativos), como elemento para la comunicación y la expresión (uso de aula virtual para la coordinación de trabajos grupales, contacto con pares y profesores, consulta de evaluaciones etc.) y como instrumento para la investigación (recurso de búsqueda y consulta de evidencias en bases de datos, uso de software de análisis de datos). Existen multitud de herramientas, y cada cual tiene sus particularidades, pero una clasificación básica en el apartado de Hardware incluye el ordenador-portátil como editor de textos, videos y realización de presentaciones tanto individuales como grupales para los alumnos; cañón o retroproyector para la proyección de videos a gran tamaño, análisis de juegos y tareas, aparato DVD/CD/MP3 utilizados principalmente para la reproducción de música. En cuanto al software podemos encontrar multitud de aplicaciones para la preparación de sesiones de Educación Física, que permiten seleccionar ejercicios para componer sesiones, disponer de una base de datos donde poder consultar juegos y actividades (Datagym, Ludos ), programas para la planificación y control del entrenamiento (EZsound, VisualFísic), programas para la evaluación (Efos). Además de todos estos recursos, no podemos olvidar las posibilidades que ofrece la red como fuente para nuestra área, utilizando buscadores especializados en educación física y deporte, foros, chats, blogs, listas de distribución, utilización de JClic, Webquest, Hotpotatoes y aplicaciones específicas interactivas como Kahoot, Plickers o Genially, que fomentan entornos de trabajo cooperativos y dinámicos.

4. Enfocar las secuencias de enseñanza-aprendizaje hacia la adquisición de competencias profesionales y el acceso a la función docente.

Como se ha mencionado anteriormente, en los actuales planes de estudio, las horas lectivas para el tratamiento de contenidos relativos a las competencias profesionales específicas de los maestros de mención son un bien escaso. Ante esta situación, parece de vital importancia que la dirección de los esfuerzos del personal docente y los planes de estudio estén bien orientados a maximizar el tratamiento de los contenidos imprescindibles y el logro de los objetivos más significativos (competencia en programación) para afrontar el reto de superar con éxito el proceso de acceso a la función docente ya sea a través de la concurrencia a oposiciones como a través de la contratación privada o concertada. Por otro lado, el acceso a la función docente solo es el principio, 
por lo que competencias como la capacidad de desarrollar los valores en el alumnado, el dominio de los contenidos didácticos objeto de estudio o la capacidad de establecer relaciones fluidas y en positivo con los padres que deriven en una alta implicación de estos en el aprendizaje de sus hijos representan talentos muy valorados por los nuevos docentes (Pesquero et al., 2008) y un reto para los formadores. El enfoque del área de Educación Física y Deportiva de la URJC, conscientes de la complejidad de esta empresa, concede gran relevancia al desarrollo de talleres sobre programación, a la resolución de situaciones teórico- prácticas con implicación de distintos agentes y miembros de la comunidad educativa, y al diseño y dirección de secuencias didácticas donde se manifieste un tratamiento específico de los contenidos propios de la Educación Física atendiendo, también, a la dimensión socioafectiva de la labor docente.

\section{Referencias}

Boud, D., Molloy E. (2015). El feedback en educación superior y profesional. Madrid: Narcea ediciones.

Cabeza Leiva, A. (2011). Individualización del proceso de enseñanza-aprendizaje. Pedagogía Magna, 11, 8-13.

Carless, J.R. (2016). Diseñar el feedback para promover el dialogo. El feedback formativo en la universidad: Experiencias con el uso de la tecnología. Barcelona. Laboratori de Mitians Interactius (LMI).

Contreras, O. (1998). Didáctica de la Educación Física: un enfoque constructivista. Barcelona: Inde.

Decreto 89/2014, de 24 de julio, del Consejo de Gobierno, por el que se establece para la Comunidad de Madrid el Currículo de la Educación Primaria.

Del Valle, S; De la Vega, R. (2007). El papel reservado al profesor de Educación Física: organizador de la interacción del alumno con el conocimiento a través del juego. EF Deportes- Revista Digital, 11(105).

Eirín, R. (2018). Las comunidades de aprendizaje como estrategia de desarrollo profesional de docentes de Educación física. Estudios Pedagógicos, 1, 259-278. https:// doi.org/10.4067/S0718-07052018000100259

Ferro, C., Martinez, M.I., Otero, M.C. (2009). Ventajas del uso de las TICs en el proceso de enseñanza-aprendizaje desde la óptica de los docentes universitarios españoles. Edutec. Revista electrónica de tecnología educativa, 29, a119. https://doi.org/10.21556/edutec.2009.29.451

García Merino, JD., Urionabarrenetxea, S. y Bañales Mallo, A (2016). Cambios en metodologías docentes y de evaluación: ¿Mejoran el rendimiento del alumnado universitario? Revista electrónica de investigación educativa, 18(3), 1-19.

Gimeno, J. y Pérez, A. (1989). La enseñanza: su teoría y su práctica. Madrid: Akal.

González Peiteado, M. (2013). Los estilos de enseñanza y aprendizaje como soporte de la actividad docente. Revista Estilos de Aprendizaje, 11(11), 51-70.

Gutiérrez, C., Hortigüela, D., Peral, Z., Pérez-Pueyo, A. (2018). Percepciones de alumnos del Grado en Maestro en Educación Primaria con Mención en Educación Física sobre la Adquisición de Competencias, Estudios Pedagógicos, 44(2), 223-239. https://doi.org/10.4067/S0718-

\section{3}

Harrow, A. (1972). A taxonomy of the psychomotor domain: a guide for developing behavioral objectives. Nueva York: David McKay Co.

Hounsell, D., McCune, V., Hounsell, J. y Litjens. J. (2008). The quality of guidance and feedback to students. Higher Education Research and Development, 27(1), 55-67.https://doi.org/10.1080/07294360701658765

Hortigüela-Alcalá, D., Pérez-Pueyo, A., López-Pastor, V. (2015). Implicación y regulación del trabajo del alumnado en los sistemas de evaluación formativa en educación superior. Relieve, 21(1). https://doi.org/10.7203/relieve.21.1.5171

Ibarra, M. y Rodríguez, G. (2007). El trabajo colaborativo en las aulas universitarias: reflexiones desde la autoevaluación. Revista de Educación, 344, 355-375.

López-Pastor, V. M., \& Pérez-Pueyo, Á. (Eds.). (2017). Evaluación formativa y compartida en educación: experiencias de éxito en todas las etapas educativas. León: Universidad de León.

Maldonado, M. (2007). El trabajo colaborativo en el aula universitaria. Laurus, 13 (23), 263-278.

Martínez Beltrán, J.M. (1995). Enseño a pensar. Madrid: Bruño.

Martínez, J. B. (2016). Crisis Económica y Financiación Educativa: Evolución de la Inversión (2009-2013). Madrid: Federación Enseñanza CC.OO.

Medina, J.L., Jarauta, B., Imbernón, F. (2010). La enseñanza reflexiva en la educación superior. Cuadernos de docencia universitaria, 17, 1- 43.

Mérida, R., Barranco, B., Criado, E., Fernández, N. López, R.M., Pérez, I. (2011). Aprender investigando en la escuela y en la universidad: una experiencia de investigación-acción a partir del Trabajo por Proyectos. Revista de Investigación en la Escuela, 73, 65-76.

Ochoa-Angrino, S., Montes-González, J., y Rojas-Ospina, T. (2018). Percepción de habilidad, reto y relevancia como predictores de compromiso cognitivo y afectivo en estudiantes de secundaria. Universitas Psychologica, 17(5), 1-18. https://doi.org/10.11144/Javeriana.upsy17-5.phrr

Pesquero, E., Sánchez, M.E., González, M., Martín, R., Guardia, S., Cervelló, J., Fernández, P., Martínez, M., Varela, P. (2008) Las competencias profesionales de los maestros de Primaria. Revista Española de Pedagogía, 66(241), 447-466.

Parellada, M. (Ed.). (2015). Informe CYD 2015. La contribución de las universidades españolas al desarrollo. Barcelona: Fundación Conocimiento y Desarrollo.

Rodriguez Sanchez, M. (2011). Metodologías docentes en el EEES: de la clase magistral al portafolio. Tendencias pedagógicas, 17, 83-103.

Vallejo, M., \& Molina, J. (2014). La evaluación auténtica de los procesos educativos. Revista Iberoamericana de educación, 64, 11-25.

Vargas, J., Chiroque, E., \& Vega, M. V. (2016). Innovación en la docencia universitaria. Una propuesta de trabajo interdisciplinario y colaborativo en educación superior. Revista de Educación, 25(48), 67-84. 